\section{The Broad's outlook in cancer}

\section{By Michael J. Haas, Senior Writer}

When the Broad Institute of MIT and Harvard launched its Center for the Science of Therapeutics to develop new therapies for hard-to-hit targets and test them in the clinic, it lacked funds to take discoveries all the way to approval. The center's new collaboration with Bayer AG fills that gap for cancer assets and comes on the heels of its infectious disease arrangement with AstraZeneca plc.

Under the five-year collaboration, the partners will explore each other's compound libraries, share screening platforms and contribute medicinal chemistry expertise to discover new small molecules for cancer.

The Broad Institute and the Center for the Science of Therapeutics (CSofT) also will contribute cancer genomics capabilities, including sequencing and computational biology tools and a deep mechanistic understanding of how mutations lead to cancer.

Although the pharma will work with researchers throughout the Broad Institutenot just at CSofT-the collaboration reflects CSofT's goal of "enabling INDs and delivering new therapies to patients, not just building a preclinical pipeline," CSofT director Stuart Schreiber told SciBX.

The Broad Institute could conduct proof-of-concept trials in small populations, but "we can't take new drug molecules through approval on our own. This requires corporate partnerships," he said.

Schreiber and Matthew Meyerson, a senior associate member at the Broad Institute, are coleading the collaboration with Bayer.

Schreiber also is director of chemical biology at the Broad Institute, a professor of chemistry and chemical biology at Harvard University and an investigator at the Howard Hughes Medical Institute. Meyerson is also a professor of pathology at Harvard Medical School and the Dana-Farber Cancer Institute.

The collaboration with Bayer is not the Broad Institute's-or CSofT's-first foray into industrial partnerships. In 2012, the institute announced a two-year deal with AstraZeneca to develop new antiviral and antibacterial therapeutics.

Earlier this year, CSofT took over the institute's role in the collaboration. CSofT is screening the institute's 100,000-compound library. Despite its relatively small size, the library is customized to contain molecular shapes and structures not found in other compound libraries, thus enabling screens of challenging targets. The pharma will optimize, develop and commercialize the hits.

Schreiber said that to identify potential partners, leaders at CSofT and the Broad Institute meet periodically with pharmas and biotechs. "With Bayer it became evident after just a single meeting-less than a year ago — that the pharma's interests were extremely well aligned" with CSofT's, he said.

"We want to select and advance projects based on human biologyin particular, the inferences about potential targets and therapeutic strategies that can be drawn from cancer genome sequencing," Schreiber said. "We will prioritize projects based on targets that are validated by genomics information and their importance to tumor maintenance, not the ease or difficulty of hitting that target or its familiarity to us and Bayer. This means we will go after targets that might be challenging, and we will rely on innovative chemistry and chemical biology to solve the hard problems those challenges present."

He noted that the goal of each project will be to test the therapeutic hypothesis of hitting the genetic target with a small molecule in the appropriate patient population.

Joint research and steering committeeseach composed of equal numbers of members from the Broad Institute and Bayer-will make decisions about the number, type and duration of projects and their development. Schreiber said that some projects are under way, but he declined to disclose details.

The Broad Institute and Bayer will share rights to any new IP generated by the collaboration, and Bayer will have the option to license IP at the preclinical stage. Schreiber declined to disclose the financial terms of the

deal.

\section{CTRP: broad-ranging resource}

Schreiber said that among the resources the partners will use to identify and validate tumor targets are The Cancer Genome Atlas (TCGA) — to which the institute contributes-and the Cancer Therapeutics Response Portal (CTRP). The latter is an online resource developed by a Broad Institute-led team that links genetic mutations in human cancer cell lines with sensitivity to small molecules.

In August, the team reported in Cell that CTRP was publicly available online and described the experiments that led to its development. ${ }^{1}$

For the Cell study, the team measured the responses of 242 human cancer cell lines representing 19 tissue types to a set of 354 small molecules that targeted a wide range of cellular processes. The team then looked for correlations between the genetic or lineage features of a cell line and its sensitivity to the small molecules.

Such relationships could be used to develop new therapeutic hypotheses and accelerate drug discovery, the team wrote in its report.

As an example of the insights CTRP could generate, the team said that it found correlations between activating mutations in $\beta$-catenin (CTNNB1) 


\section{ANALYSIS}

\section{TRANSLATIONAL NOTES}

and sensitivity to navitoclax in human colorectal, endometrial, gastric, liver and lung cancer cell lines. The study also revealed correlations between sensitivity to navitoclax and loss-of-function mutations in AXIN1 and casein kinase $1 \alpha(C S N K 1 A 1 ; C K I-\alpha)$, which play roles in CTNNB1 degradation.

Thus, mutations in CTNNB1, AXIN1 or CSNK1A1 could help identify tumors that would respond to navitoclax and other inhibitors of $\mathrm{B}$ cell lymphoma 2 (BCL-2; BCL2) family proteins, the team wrote.

AbbVie Inc. and Roche's Genentech Inc. unit have navitoclax (ABT-263; RG7433), a pan-inhibitor of antiapoptotic members of BCL2 family proteins, in Phase I/II testing for small cell lung cancer and Phase I trials for solid tumors.

Paul Clemons, Alykhan Shamji and Schreiber co-led the team. Clemons is director of computational chemical biology research at Csof T, and Shamji is the center's executive director. The team included researchers from Columbia University and the Vanderbilt University School of Medicine.

Data from the study are publicly available online at the Cancer Therapeutics Response Portal.

Clemons said that the team's ongoing work includes testing navitoclax in mice with CTNNB1-mutant xenograft tumors and expanding CTRP with additional cancer cell lines, drug compounds and online functionalities.

The findings reported in Cell are unpatented and unlicensed, he said.

Last week, a separate Broad Institute team reported in Nature Genetics the results of a study that analyzed patterns and functional consequences of somatic copy number alterations (SCNAs) in over 4,900 cancers from TGCA. ${ }^{2}$

A key finding of the study was that SCNAs tend to reside in just 140 regions of the genome, only 35 of which contained known oncogenes or tumor suppressor genes. The team said that future studies need to identify whether the remaining regions encompass additional oncogenes or tumor suppressors or have limited functional roles in tumorigenesis.

Haas, M.J. SciBX 6(38); doi:10.1038/scibx.2013.1051

Published online Oct. 3, 2013

\section{REFERENCES}

1. Basu, A. et al. Cell; published online Aug. 29, 2013; doi:10.1016/j.cell.2013.08.003

Contact: Stuart L. Schreiber, Broad Institute of MIT and Harvard, Cambridge, Mass.

e-mail: stuart_schreiber@harvard.edu

Contact: Alykhan F. Shamji, same affiliation as above e-mail: ashamji@broadinstitute.org Contact: Paul A. Clemons, same affiliation as above e-mail: pclemons@broadinstitute.org

2. Zack, T.l. et al. Nat. Genet. 45, 1134-1140 (2013)

\section{COMPANIES AND INSTITUTIONS MENTIONED}

AbbVie Inc. (NYSE:ABBV), Chicago, III. AstraZeneca plc (LSE:AZN; NYSE:AZN), London, U.K. Bayer AG (Xetra:BAYN), Leverkusen, Germany Broad Institute of MIT and Harvard, Cambridge, Mass. Columbia University, New York, N.Y.

Dana-Farber Cancer Institute, Boston, Mass. Genentech Inc., South San Francisco, Calif. Harvard Medical School, Boston, Mass. Harvard University, Cambridge, Mass. Howard Hughes Medical Institute, Chevy Chase, Md. Roche (SIX:ROG; OTCQX:RHHBY), Basel, Switzerland Vanderbilt University School of Medicine, Nashville, Tenn. 